\title{
Dynamic SLA Negotiation in Autonomic Federated Environments
}

\author{
Pawel Rubach ${ }^{1,2}$ and Michael Sobolewski ${ }^{1}$ \\ ${ }^{1}$ Computer Science, Texas Tech University, SORCER Research Group, \\ Box 43104 Boston \& 8th, Lubbock, TX 79409, USA \\ \{pawel.rubach, sobol\} asorcersoft.org \\ ${ }^{2}$ Business Informatics, Warsaw School of Economics, \\ Al. Niepodleglosci 162, 02-554 Warszawa, Poland
}

\begin{abstract}
Federated computing environments offer requestors the ability to dynamically invoke services offered by collaborating providers in the virtual service network. Without an efficient resource management that includes Dynamic SLA Negotiation, however, the assignment of providers to customer's requests cannot be optimized and cannot offer high reliability without relevant SLA guarantees. We propose a new SLA-based SERViceable Metacomputing Environment (SERVME) capable of matching providers based on QoS requirements and performing autonomic provisioning and deprovisioning of services according to dynamic requestor needs. This paper presents the SLA negotiation process that includes ondemand provisioning and uses an object-oriented SLA model for large-scale serviceoriented systems supported by SERVME. An initial reference implementation in the SORCER environment is also described.
\end{abstract}

Keywords: SLA Negotiation, QoS, SLA, Metacomputing, Service-Oriented Architecture, SORCER.

\section{Introduction}

Many research activities worldwide are focused on developing smart, selfmanageable systems that will allow applications to run smoothly and reliably in a distributed environment. IBM calls this Autonomic Computing [1]. The realization of this concept would enable the move towards Utility Computing - the long awaited vision where computing power would be available as a utility just like water or electricity is delivered to our homes today. One of the challenges in addressing this concept lies in the problem of guaranteeing a certain level of Quality of Service (QoS) to the customer for which he/she would be willing to pay.

In this paper we address related issues by proposing the Dynamic SLA Negotiation process for the SERViceable Metacomputing Environment (SERVME)[2] which is based on the SORCER (Service-Oriented Computing EnviRonment) [3] environment extended by adding a QoS Management Framework. This paper presents the SLA negotiation process including the on-demand provisioning of services and briefly describes the architecture of the federated P2P environment.

SORCER provides a way of creating service-oriented programs and executing them in a metacomputing environment. The service-oriented paradigm is a distributed 
computing concept wherein objects across the network play their predefined roles as service providers. Service requestors can access these providers by passing messages called service exertions. An exertion defines how the service providers federate among themselves to supply the requestor with a required service collaboration. All these services form an instruction-set of a virtual metacomputer that looks to the enduser as a single computer.

The proposed SLA negotiation process has been implemented and validated as part of the SERVME framework in the SORCER environment. However, due to its generic nature we believe that both the Service Level Agreements (SLA) object model as well as the underlying communication model defined in terms of communication interfaces could be adopted for other service-oriented architectures.

This paper is a follow-up to [2] - the first one to describe the SERVME framework. Here the focus is the SLA life-cycle and negotiation whereas [2] concentrated on the SERVME architecture and the SLA object model.

The rest of the paper is divided into the following sections: Section 2 describes the related work, Section 3 gives introduction to SORCER, Section 4 presents the overview of SERVME, Section 5 elaborates on the SLA negotiation, Section 6 presents the deployment of the framework, and Section 7 concludes the paper.

\section{Related Work}

SLA negotiation has been researched extensively at first in the area of networking. Its application to services was propagated with the emergence of Grid Computing. At first the Globus Resource Allocation Manager (GRAM) [4] lacked a general negotiation protocol that was added later (as described in [5]) in form of the Service Negotiation and Acquisition Protocol (SNAP) [6] that addresses complex, multi-level SLA management. SNAP defines three types of SLAs: Task SLAs, Resource SLAs and Binding SLAs and provides a generic framework, however as Quan et al. [7] underline the protocol needs further extensions for its implementation to address specific problems.

As grid technology started to move from traditional network batch queuing towards the application of Web Services (WS) the work of the grid community as well as others focused on incorporating SLA negotiation into the stack of WS technologies. The Web Service Level Agreement framework (WSLA) [8] and the WS-Agreement specification [9] have been proposed to standardize the SLA specification. WS-Agreement specifies also basic negotiation semantics, however, allows only a simple one-phase - offer-accept/reject negotiation. More complex twoand three-phase commit protocols applied in conjunction with WS-Agreement are described in [10]. A different approach to enable automatic SLA negotiation was taken by [11] and [12] who propose to use agents for the negotiation of SLAs in grids. In [13] authors propose to introduce a meta-negotiation protocol that will allow the parties to select via negotiation the protocol used for the actual SLA negotiation.

The above solutions concentrate on traditional grids or WS architectures, however, new challenges that reach beyond the multi-phase commit protocols arise when introducing P2P resource management. Significant work has also been pursued in this area, for example by [15], however, this research does not include SLA negotiation. 
A novel approach to SLA management and negotiation for P2P distributed environments where federations of services are formed on-the-fly is presented. To fully address the problems with network/resource unreliability and contract SLAs for multi-level, multiple party scenarios this paper introduces a leasing mechanism that is used in conjunction with the 2-phase commit transactional semantics.

\section{SORCER}

SORCER [3] is a federated service-to-service (S2S) metacomputing environment that treats service providers as network objects with well-defined semantics of a federated service object-oriented architecture. It is based on Jini [16] semantics of services in the network and Jini programming model with explicit leases, distributed events, transactions, and discovery/join protocols. While Jini focuses on service management in a networked environment, SORCER focuses on exertion-oriented programming and the execution environment for exertions [3]. SORCER uses Jini discovery/join protocols to implement its exertion-oriented architecture (EOA) [18], but hides all the low-level programming details of the Jini programming model.

In EOA, a service provider is an object that accepts remote messages from service requestors to execute collaboration. These messages are called service exertions and describe collaboration data, operations and collaboration's control strategy. An exertion task (or simply a task) is an elementary service request, a kind of elementary instruction executed by a single service provider or a small-scale federation for the same service data. A composite exertion called an exertion job (or simply a job) is defined hierarchically in terms of tasks and other jobs, a kind of federated procedure executed by a large-scale federation. The executing exertion is dynamically bound to all required and currently available service providers on the network. This collection of providers identified in runtime is called an exertion federation. The federation provides the virtual processor (metaprocessor) for the collaboration as specified by its exertion. When the federation is formed, each exertion's operation has its corresponding method (code) available on the network. Thus, the network exerts the collaboration with the help of the dynamically formed service federation. In other words, we send the request onto the network implicitly, not to a particular service provider explicitly.

The overlay network of service providers is called the service grid and an exertion federation is in fact a virtual metaprocessor. The metainstruction set of the metaprocessor consists of all operations offered by all providers in the service grid. Thus, an exertion-oriented (EO) program is composed of metainstructions with its own control strategy and a service context representing the metaprogram data. These operations can be specified in the requestor's exertion only, and the exertion is passed by itself on to the initializing service provider (found dynamically) via the top-level servicer interface implemented by all service providers called servicers-service peers. Thus all service providers in EOA implement the service(Exertion, Transaction) : Exertion operation of the Servicer interface.

Domain specific servicers within the federation, or task peers (taskers), execute task exertions. Rendezvous peers (jobbers and spacers) coordinate execution of job exertions. Providers of the Tasker, Jobber, and Spacer type are three of SORCER main infrastructure servicers. 
To further clarify what an exertion is, an exertion consists mainly of three parts: a set of service signatures, which is a description of operations in collaboration, the associated service context upon which to execute the exertion, and control strategy (default provided) that defines how signatures are applied in the collaboration. A service signature specifies at least the provider's service type (interface) that the service requestor would like to use and a selected operation to run within that interface. A service context consists of several data nodes used for either: input, output, or both. A task works with only a single service context, while a job may work with multiple service contexts since it can contain multiple tasks [18].

In SERVME a signature includes a QoS Context (described in Section 4.2) that encapsulates all QoS/SLA data.

\section{SERVME Overview}

To perform SLA negotiation one has to define: 1) a SLA negotiation protocol and interactions between components, 2) a QoS/SLA specification and 3) a negotiation strategy or a decision-making model. SERVME defines the negotiation protocol in the form of a generic communication model, its components tailored to the requirements of federated environments as well as the SLA specification in form of an object model and its data structures. A default negotiation strategy and a decisionmaking model is presented below, however, SERVME is designed to allow an easy customization of the negotiation business logic for each provider and requestor since in a real-world scenario of a free-market service economy these rules may decide which provider receives more requests and thus may become part of its competitive advantage and be considered confidential.

\subsection{SERVME Components}

SERVME builds on the SORCER environment by extending its interfaces and adding new service providers. The details of the architecture have been described in [2]. The components used in the SLA negotiation process are shortly presented below.

- Serviceprovider provides the requested service and has a built-in component called SlaDispatcher that retrieves the QoS parameters from the operating system and is responsible for the SLA management on the provider side.

- QosCatalog is an independent service that acts as an extended Lookup Service (QoS LUS) as well as the SLA negotiation broker between the provider and the requestor.

- Slaprioritizer is a component that allows controlling the prioritization of the execution of exertions according to organizational requirements (see section 4.2)

- SlaMonitor is an independent service that acts as a registry for negotiated SLA contracts and exposes the user interface (UI) for administrators to allow them to monitor and cancel active SLAs.

- OnDemandProvisioner is a SERVME provider that enables on-demand provisioning of services in cooperation with the Rio Provisioner [14] [16]. The Qoscatalog uses it when no matching service provider can be found that meets requestor QoS requirements. 


\subsection{SLA Object Model}

The key feature of the framework is the proposed SLA object model designed to meet the requirements of federated metacomputing environments. For a detailed description including a UML class diagram please refer to [2].

The two main artifacts are: QosContext and SlaContext. The first one groups the requirements submitted by the requestor. It contains: 1) Functional Requirements - a service type (interface) identifying a requested provider, operation to be executed, and related provider's attributes, 2) System Requirements-fixed properties that describe the requested provider's hardware and software environment (i.e. CPU architecture, OS name and version etc.), 3) Organizational Requirementsproperties of the submitting entity (department, team, project, requested timeframe for the execution, priority etc.), 4) Metrics-dynamic, user defined, compound parameters which are calculated on the basis of System- or Organizational Requirements, 5) Service Cost-requirements (i.e. Maximum cost of the execution) and 6) SLA Parameter Requests - the demanded ranges of values or fixed values of QoS parameters.

The second critical interface-Slacontext defines the actual SLA. It contains the related requirements in form of the QosContext as well as: 1) SLA Parameters offered or guaranteed by the provider 2) the offered price 3 ) data used to identify the provider (its ID, proxy etc.) and 4) the state of the negotiation that can have one of the enumerated values: SLA_REQUESTED，SLA_UPDATED，SLA_OFFERED，SLA_ACCEPTED，SLA_GRANTED， SLA_ARCHIVED.

\section{SLA Negotiation}

This section describes the SLA negotiation process. Fig. 1 shows how the negotiation process is integrated into the life-cycle of executing exertions. The diagram refers also to other two activity diagrams presented below: SLA Negotiation and SLA Monitoring.

\subsection{Recursive Acquisition of SLAs}

The negotiation sequence for a single exertion of Task type is presented below in detail, however for completeness in this subsection more complex exertions that require recursive SLA acquisition are shortly described.

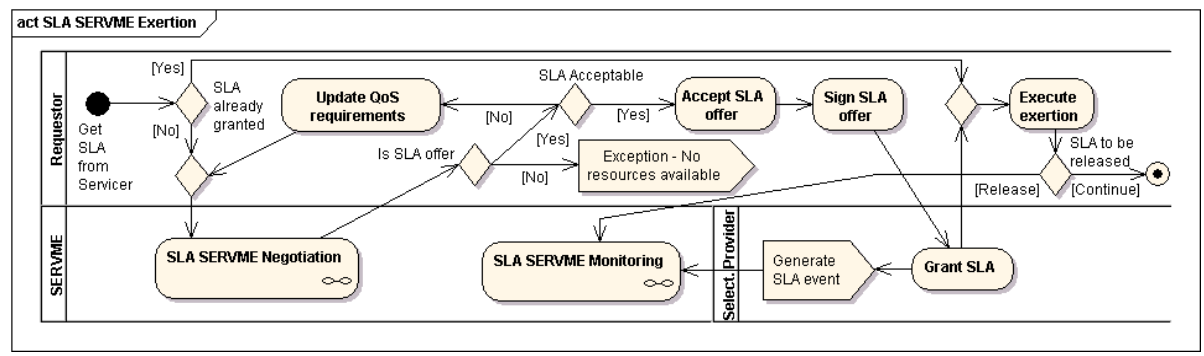

Fig. 1. Activity Diagram showing how SERVME SLA Negotiation is integrated into EOP 
The execution of an exertion begins when the requestor calls Exertion.exert (). In case the exertion is of Task type the request is passed on to the Qoscalatog that acts as a broker in the negotiation process described below. However, if the exertion is of Job type, then QosCalatog finds in runtime a matching rendezvous provider (Jobber or Spacer) with a guaranteed SLA.

Before the guaranteed SLA is returned, the rendezvous provider recursively acquires SLAs for all component exertions as described below depending on the type (Task or Job) of component exertion. To ensure transactional semantics of the SLA acquisition the rendezvous peer uses a leasing mechanism (described below) that is similar to the two-phase commit protocol defined by the Jini Transaction model.

Exertions of Task type may also contain multiple signatures (as explained in Section 3), so the same recursive mechanism is used to acquire the final SLA. However, in this case the requestor only receives the final SLA for the dynamically binding - signature of the PROCESS type.

For intelligibility in the following subsections the assumption is that the outcome of the negotiation should be a single SLA contract for a Task with only one signature.

\subsection{Preliminary Selection of Providers}

As depicted in Fig. 2 at first QosCatalog analyzes the QoS requirements passed in the Qoscontext and extracts the functional requirements (provider's interface, method, and other attributes) as well as system requirements. Based on the functional

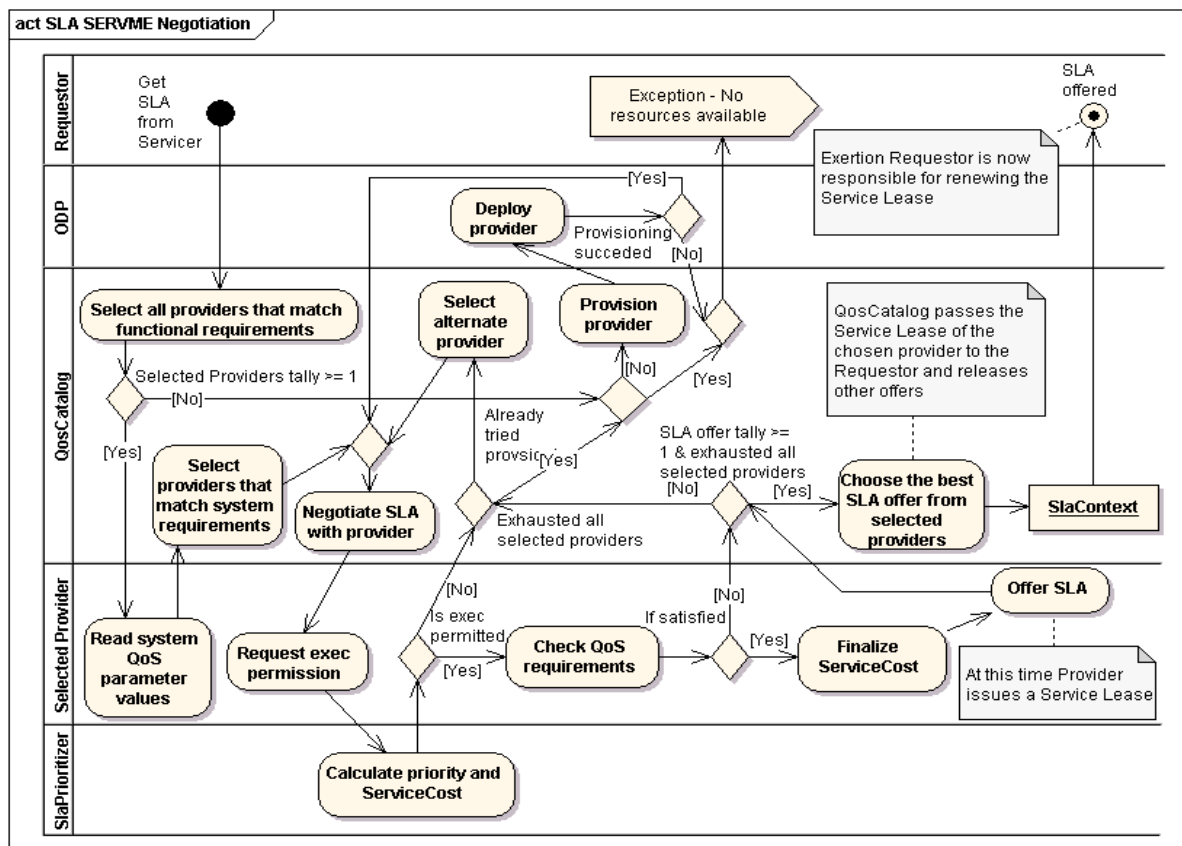

Fig. 2. Activity Diagram showing the actual SLA Negotiation 


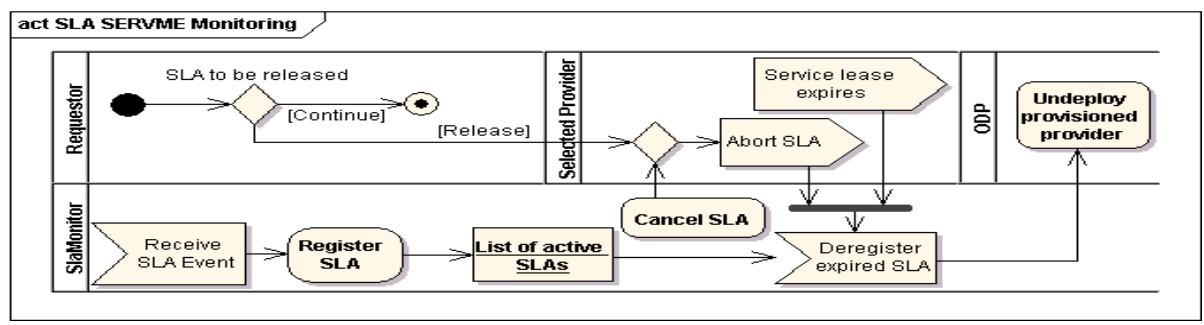

Fig. 3. Activity Diagram showing the SLA Monitoring

requirements QosCatalog performs a dynamic lookup and retrieves a list of all providers offering the requested interface and method. If none are found QosCatalog tries to provision them using the OnDemandProvisioner (ODP) (see subsection 5.5). Next, QosCatalog queries the ServiceProvider to retrieve the basic QoS parameters that it can offer. The supplied data allows it to select providers that match the system requirements. Those are then called via their SlaManagement interface to start the SLA negotiation process.

\subsection{Negotiation}

The negotiation is initiated by the Qoscatalog that invokes the negotiatesla operation of the SlaManagement interface of the provider. In the first step the provider extracts the organizational requirements from the Qoscontext and passes them to the Slaprioritizer where the exertion's organizational properties are evaluated against strategic rules defined by the management in the SlaPrioritizer service. The provider then receives a permission or denial to execute the exertion and optionally a cost parameter that it may use to calculate the final service cost of the offer. In case no permission is given the provider returns a no-go exception and the Qoscatalog has to select an alternate provider or autonomically provision one if no others are available. After locating another provider the negotiation sequence is repeated for that provider.

In case the permission is given the provider checks the QoS requirements against its current resource utilization and allocations for other concurrently guaranteed SLAs. If a parameter can be guaranteed the provider copies the corresponding Slaparameter object including the requested threshold values from the QosContext's SLA parameter requests to SlaContext's SLA parameters and sets its state to PARAM_OFFERED. However, if the requirement cannot be fulfilled the corresponding SLA parameter request is also copied to SlaContext but its state is set to PARAM_UPDATE and its threshold range is updated to the maximum/minimum offered value. After processing individual parameters the provider sets the state of the whole Slacontext to SLA_OFFERED if all SLA parameters can be guaranteed or SLA_UPDATED otherwise.

In case the QoS requirements can be met the provider calculates the estimated service cost, allocates the offered resources and creates a Lease that is attached to the SLA offer. This Lease has a short expiration time and thus guarantees that the resources are not blocked unnecessarily. Before the exertion is finally executed the 
Lease must be renewed by the requestor to extend the life of the SLA. The estimated cost in the validation case, for example, is calculated on the basis of historical executions with similar input data on the same host. Cost is inversely proportional to time of execution extended with some parameters that altogether causes that running computations on faster hardware is much more expensive than on lower-end hosts.

To guarantee the non-repudiation of contracts or offers the provider uses the SORCER security framework based on PKI infrastructure to sign the SLA offer before passing it on to the QosCatalog.

The described negotiation sequence is repeated by the Qoscatalog for all providers that initially matched the system requirements. Out of all offers the QosCatalog chooses the best one depending on the specified parameters and passes it to the requestor for acceptance and signing (see Fig. 1). Currently time only or cost only optimizations are supported but the inclusion of non-linear optimization methods that will allow to select a set of offers matching both parameters (i.e., fastest execution but costing no more than $\mathrm{X}$ ) are a work in progress.

\subsection{SLA Acceptance and Signing}

The requestor may now decide to accept or deny the received offer. However, in case it is denied the SLA negotiation process has to be reinitiated from the very beginning. In case of acceptance the requestor updates the SLA's state to SLA_ACCEPTED and performs digital signing using the PKI infrastructure.

From now on the requester is responsible for renewing the Lease of the SLA.

The requester calls the signsla method of the provider and passes the Slacontext. If the Lease has not expired the provider grants the SLA by setting its state to SLA_GRANTED. The Slacontext is then returned to the requestor and the execution of the exertion may finally begin.

At the same time the provider sends a copy of the Slacontext asynchronously to the SlaMonitor where it is registered and persisted.

\subsection{On-Demand Provisioning}

SERVME reduces the overall resource utilization by allowing service providers to be provisioned on-demand and deprovisioned when they are not used anymore.

In the above negotiation process there are three scenarios that may lead to ondemand provisioning: 1) when no providers are available that meet functional requirements 2) when none of the available providers receive a permission to execute the exertion from the SlaPrioritizer and 3) when none of the SLA offers returned by providers to the Qoscatalog fully fulfills the requirements (all have a state of negotiation set to SLA_UPDATED).

In any of these cases the Qoscatalog tries to deploy a new provider with the required QoS parameters by calling the OnDemandProvisioner object. OnDemandProvisioner constructs on-the-fly an Operationalstring required by Rio and calls the ProvisionMonitor component of Rio [14] to deploy the required providers. If the provisioning succeeds Qoscatalog invokes the same negotiation sequence on the newly provisioned provider. Otherwise QosCatalog returns to the 
requestor the full list of SLAs that it negotiated, none of which however, fully fulfills the requestors requirements. The requestor may now choose to accept one of these offers or try to start another round of negotiation with lowered QoS requirements.

\subsection{SLA Monitoring and Management}

As depicted in Fig. 3 the SlaMonitor can be used to monitor the execution and delete an active SLA. It communicates with providers and asynchronously receives messages with updated states of the SLA's lifecycle.

\subsection{Deprovisioning Services}

The leasing mechanism described in subsection 5.3 ensures that the provider is aware when any of the granted SLAs expires or the exertion simply finishes execution. This information is passed on to the SlaMonitor that also receives events regarding the provisioning actions taken by the OnDemandProvisioner. SlaMonitor is thus able to detect situations when the provisioned provider is not used anymore. In that case it notifies the OnDemandProvisioner and this service undeploys the unused provider by calling the Rio's ProvisionMonitor. The provider cannot just simply destroy itself upon finishing the execution of the exertion since in that case Rio's failover mechanism would immediately deploy another instance of that provider.

\section{Deployment}

SERVME has been deployed in the SORCER environment. The framework was validated in a real-world example taken from neuroscience. SERVME was used to invoke and control multiple parallel and sequential computations that dealt with the processing of MRIs of human brains. Six heterogeneous hosts where used to perform several simultaneous computations. The simulations were run several times and have shown that with SERVME it is possible to optimize the execution of complex computations for lowest price or best performance. The overhead time resulting from the communication needed to select the appropriate provider, performing SLA negotiation, and signing the SLA contract has been measured in this environment at around 1-1.5 seconds and as such is negligible in comparison to the computations run, that took minimally 3-4 minutes each. Detailed validation results along with a complete statistical analysis will be published in a forthcoming paper.

\section{Conclusions}

The new SLA Negotiation process for Autonomic Federated Metacomputing Environments is presented in this paper. The described process includes the ondemand provisioning of services and refers to components defined in the SERVME framework: Qoscatalog, SlaDispatcher, SlaMonitor, SlaPrioritizer, and OnDemandProvisioner. The negotiation uses the SLA object model introduced in SERVME and defined by the two generic interfaces: QosContext and related slacontext. To the best of our knowledge this is the first attempt to describe the SLA negotiation process for exertion-oriented programming. 
The presented framework addresses the challenges of spontaneous federations in SORCER and allows for better resource allocation. Also, SERVME provides for better hardware utilization due to Rio monitored provisioning and SORCER ondemand provisioning. The presented architecture scales very well with on-demand provisioning that reduces the number of compute resources to those presently required for collaborations defined by corresponding exertions. When diverse and specialized hardware is used, SERVME provides means to manage the prioritization of tasks according to the organization's strategy that defines "who is computing what and where".

Two zero-install and friendly graphical user interfaces attached to SLA Monitor and SORCER Servicer are available for administration purposes.

The SERVME providers are SORCER Servicers so additional SERVME providers can be dynamically provisioned if needed autonomically. Finally, the framework allows for accounting of resource utilization based on dynamic cost metrics, thus it contributes towards the realization of the utility computing concept.

Acknowledgments. This work was partially supported by Air Force Research Lab, Air Vehicles Directorate, Multidisciplinary Technology Center, the contract number F33615-03-D-3307, Service-Oriented Optimization Toolkit for Distributed High Fidelity Engineering Design Optimization. We would like also to thank Dennis Reedy the architect of project Rio for his invaluable assistance that helped us to integrate the Rio provisioning framework with the SERVME framework.

\section{References}

[1] Kephart, J.O., Chess, D.M.: The vision of autonomic computing. Computer 36, 41-50 (2003)

[2] Rubach, P., Sobolewski, M.: Autonomic SLA Management in Federated Computing Environments. In: Proceedings of the 2009 International Conference on Parallel Processing Workshops (ICPPW 2009). IEEE Computer Society, Los Alamitos (in press, 2009)

[3] Sobolewski, M.: SORCER: Computing and Metacomputing Intergrid. In: 10th International Conference on Enterprise Information Systems, Barcelona, Spain (2008)

[4] Czajkowski, K., Foster, I., Karonis, N., Kesselman, C., Martin, S., Smith, W., Tuecke, S.: A resource management architecture for metacomputing systems. In: Feitelson, D.G., Rudolph, L. (eds.) IPPS-WS 1998, SPDP-WS 1998, and JSSPP 1998. LNCS, vol. 1459, pp. 62-82. Springer, Heidelberg (1998)

[5] Czajkowski, K., Foster, I., Kesselman, C., Tuecke, S.: Grid service level agreements: Grid resource management with intermediaries. In: Grid resource management: state of the art and future trends, pp. 119-134. Kluwer Academic Publishers, Dordrecht (2004)

[6] Czajkowski, K., Foster, I., Kesselman, C., Sander, V., Tuecke, S.: SNAP: A Protocol for Negotiating Service Level Agreements and Coordinating Resource Management in Distributed Systems. In: Feitelson, D.G., Rudolph, L., Schwiegelshohn, U. (eds.) JSSPP 2002. LNCS, vol. 2537, pp. 153-183. Springer, Heidelberg (2002)

[7] Quan, D.M., Kao, O.: SLA Negotiation Protocol for Grid-Based Workflows. In: High Performance Computing and Communcations, pp. 505-510 (2005) 
[8] Ludwig, H., Keller, A., Dan, A., King, R.P., Franck, R.: Web service level agreement (WSLA) language specification. IBM Corporation (2003)

[9] Andrieux, A., Czajkowski, K., Ibm, A.D., Keahey, K., Ibm, H.L., Nec, T.N., Hp, J.P., Ibm, J.R., Tuecke, S., Xu, M.: Web Services Agreement Specification, WS-Agreement (2007)

[10] Pichot, A., Wieder, P., Waeldrich, O., Ziegler, W.: Dynamic SLA-negotiation based on WS-Agreement, CoreGRID Technical Report TR-0082, Institute on Resource Management and Scheduling (2007)

[11] Shen, W., Li, Y.: Adaptive negotiation for agent-based grid computing. In: Proceedings of the Agentcities/Aamas 2002, vol. 5, pp. 32-36 (2002)

[12] Ouelhadj, D., Garibaldi, J., MacLaren, J., Sakellariou, R., Krishnakumar, K.: A Multiagent Infrastructure and a Service Level Agreement Negotiation Protocol for Robust Scheduling in Grid Computing. In: Sloot, P.M.A., Hoekstra, A.G., Priol, T., Reinefeld, A., Bubak, M. (eds.) EGC 2005. LNCS, vol. 3470, pp. 651-660. Springer, Heidelberg (2005)

[13] Brandic, I., Venugopal, S., Mattess, M., Buyya, R.: Towards a Meta-Negotiation Architecture for SLA-Aware Grid Services, Technical Report, GRIDS-TR-2008-9, Grid Computing and Distributed Systems Laboratory, The University of Melbourne, Australia (2008)

[14] Project Rio, http: / / rio.dev.java.net/ (accessed on March 13, 2009)

[15] Cao, J., Kwong, O.M.K., Wang, X., Cai, W.: A peer-to-peer approach to task scheduling in computation grid. Int. J. Grid Util. Comput. 1, 13-21 (2005)

[16] Jini architecture specification, Version 2.1 (accessed on March 2009)

[17] Sobolewski, M.: Federated Method Invocation with Exertions. In: Proceedings of the 2007 IMCSIT Conference, pp. 765-778. PTI Press (2007)

[18] Sobolewski, M.: Exertion Oriented Programming. In: IADIS, vol. 3, pp. 86-109 (2008) 